\title{
Cycle biologique de Tarsubulura perarmata
}

\author{
(Ratzel, 1868)
}

\author{
par J.-C. QUENTIN *, M. KRISHNASAMY * et R. TCHEPRAKOFF * \\ * Laboratoire de Zoologie (Vers), associé au C.N.R.S., \\ Muséum national d'Histoire naturelle, 43, rue Cuvier, F 75231 Paris Cedex 05, \\ et * Laboratoire de Biologie, Faculté des Sciences et Techniques Sfax, Tunisie. \\ ** Division of Medical Ecology (Lim Boo Liat). Institute for Medical Research, \\ Kuala Lumpur, Malaysia.
}

\begin{abstract}
Résuméé.
Tarsubulura perarmata (Ratzel, 1868) est identifié en Malaisie (Kuala Lumpur) chez un: Primate Tarsius bancanus, et chez des Tupaidés Tupaia glis et $T$. minor. Son cycle biologique est obtenu par l'infestation expérimentale de Criquets appartenant aux genres Valanga et Oxva. Les larves infestantes sont obtenues après trois semaines d'évolution à $28^{\circ} \mathrm{C}$ chez l'insecte. Elles diffèrent des troisièmes stades larvaires de Subulurinae par le développement des lobes pharyngiens cuticulaires. L'apparition précoce de ce caractère ontogénétique confirme l'isolement du genre Tarsubulara par rapport à l'évolution générale des Subuluridae.
\end{abstract}

\section{Summary.}

Biological cycle of Tarsubulura perarmata (Ratzel, 1868).

Tarsubulura perarmata (Ratzel, 1868) is described from a primate Tarsius bancanus and from Tupaidae: Tupaia glis and T. minor in Malaysia, (Kuala Lumpur). Its biological cycle is done by the experimental infestation of crickets belonging to the genera Valanga and Oxya. The infective larvae are obtained after three weeks of development at $28{ }^{\circ} \mathrm{C}$ in the intermediate host. They differ from third stage larvae obtained from Subulurinae by the development of cuticular pharyngeal lobes. The early apparition of this ontogenetic character confirms the isolation of the genus Tarsubulura as compared to the general evolution of the Subuluridae.

Accepté le 25 octobre 1976. 


\section{Introduction}

Tarsubulura perarmata (Ratzel, 1868) est un Nématode Subulure parasite de Primates primitifs. Cette espèce a été récoltée à Ceylan chez Tarsius spectrum. Elle est décrite chez Loris lydekkerianus par Meyer en 1896 sous le nom de Filaria sarasinorum dont la synonymie avec $T$. perarmata est indiquée par Baylis, 1944 . De la même façon l'espèce Subulura indica Khéra, 1956, décrite chez le même hôte est placée en synonymie de $T$. perarmata par Inglis, 1958.

A Sarawak (Bornéo) Baylis, 1926, récolte $T$. perarmata chez Tarsius spectrum. Dans la région de Kuala Lumpur (Malaisie) nous avons identifié ce Subulure chez Tarsius bancanus $\left(\mathrm{n}^{\circ}\right.$ d'enregistrement Division Med. Ecol. R. 91296) et aussi chez des Tupaidés Tupaia glis ( $\mathrm{n}^{\circ}$ d'enregistrement M.N.H.N. Kl 123, Kl 124, Kl 162. $\mathrm{K} 1$ 256, Kl 263) et Tupaia minor (K1 126).

Tarsubulura perarmata est un Subulure de petite taille (longueur des mâles 6$7 \mathrm{~mm}$, longueur des femelles 8-10 mm), qui se différencie aisément des autres Subuluridae par ses structures céphaliques et génitales.

Ses structures céphaliques ont la particularité de présenter deux cavités superposées nettement distinctes. Une partie buccale antérieure ( $=$ cheilostome sensu Inglis, 1966) dont les parois cuticulaires sont peu épaisses et une partie pharyngienne postérieure (= œsophastome sensu Inglis, 1966) plus profonde dont les parois sont développées (fig. 1, A).

Chez $T$. perarmata la paroi de cette cavité pharyngienne est cuticulaire. Elle est selon Inglis, 1958, constituée par les lobes pharyngiens périphériques ; les lobes chordaux et radiaux ne sont pas tordus en hélice mais indépendants les uns des autres et dressés verticalement dans la cavité buccale.

Ses structures génitales sont également remarquables par la torsion des spicules (fig. 1, E).

L'hypertélie des structures cuticulaires céphaliques et la spécialisation des structures génitales confèrent à ce parasite une morphologie archaïque. Ses hôtes Tarsiidae, Lorisiidae et Tupaidae sont d'ailleurs primitifs.

Inglis (1958) a rapproché le genre Tarsubulura du genre Labiobulura parasite de Marsupiaux australiens et isolé (1960) une lignée propre au genre Tarsubulura. L'un de nous (Quentin, 1969) dans un essai d'interprétation phylogénique des Subuluridae, a proposé de réunir les deux genres Tarsubulura et Labiobulura au sein d'une même sous-famille, celle des Labiobulurinae et émis l'hypothèse que ce groupe zoologique se serait détaché très tôt dans la phylogénie des Subuluridae.

Sur le plan de la biologie larvaire, deux éléments présentent un intérêt systématique chez les Subuluridae.

La croissance larvaire aboutit dans le genre primitif Allodapa parasite d'Oiseaux à une larve infestante inférieure à $500 \mu$. Cette croissance est par contre plus impor- 
tante chez les Subulures parasites de Mammifères dont les cycles sont connus (genres Subulura, Primasubulura), la larve infestante ayant une taille toujours supérieure à 1000 u.
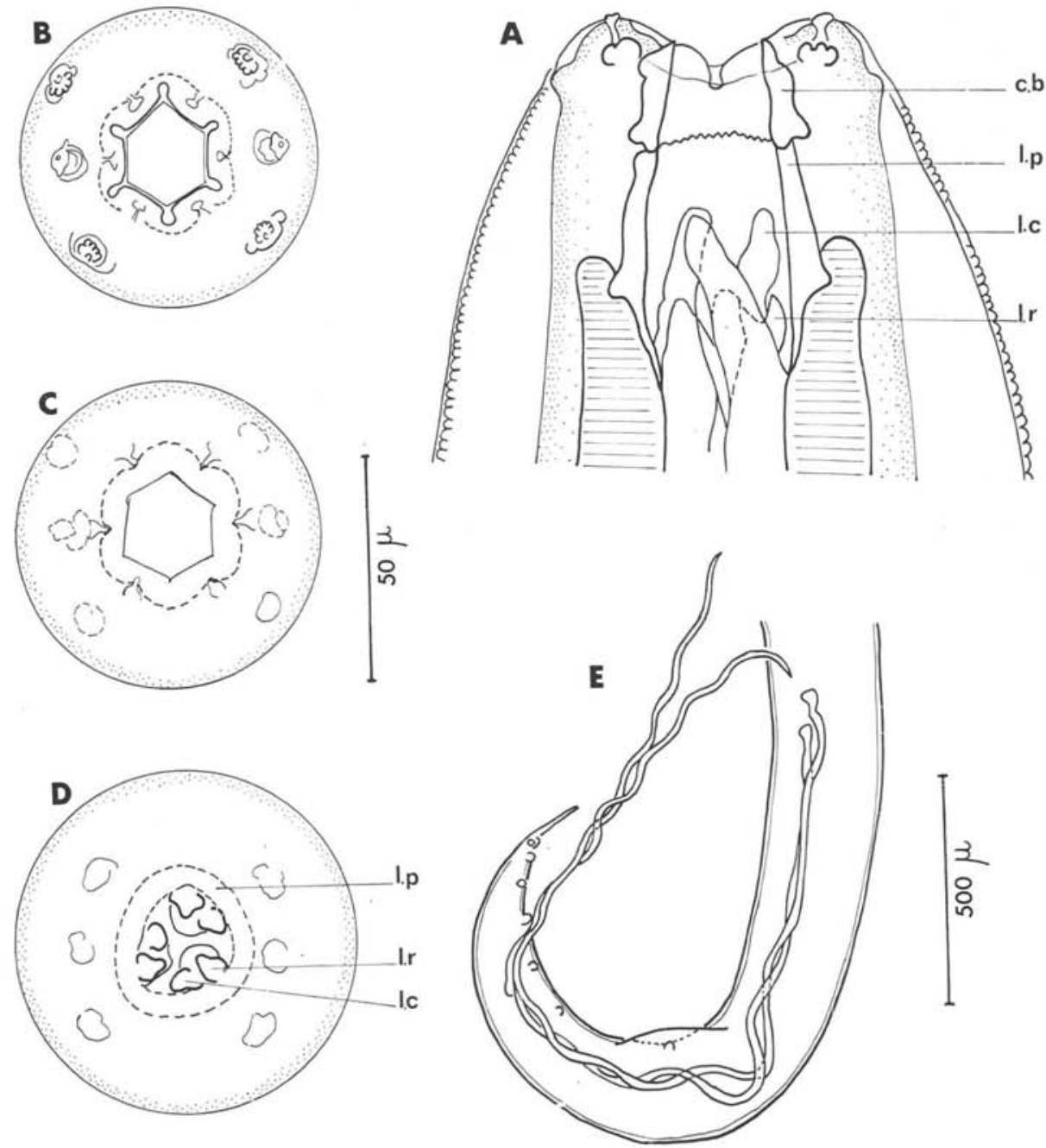

Fig. 1. - Tarsubulura perarmata. A: extrémité céphalique, adulte, vue dorsale. c.b. : paroi de la cavité buccale ; 1.p. : lobe périphérique ; 1.c. : lobe chordal ; 1.r. : lobe radial: B : tête de l'adulte, vue apicale au niveau de la bouche; C: coupe optique de la capsule buccale dans la première portion; $\mathrm{D}$ : coupe à la base de la capsule buccale au niveau des lobes pharyngiens; E: bourse caudale du mâle, en vue latérale, avec spicules de forme hélicoïdale.

A, B, C, D : éch. $50 \mu$; E : éch. $500 \mu$. 
Les structures céphaliques des larves du troisième stade sont dans ces deux groupes toutes identiques et comparables à celles des adultes du genre Allodapa. L'évolution de ces structures s'observe chez les formes les plus évoluées du troisième stadc à l'adulte. Elle permet de classer les Subulures à leur place zoologique respective, selon leur degré de différenciation céphalique.

Nous étudions le cycle évolutif de Tarsubulura perarmata en nous appuyant principalement sur ces éléments: croissance larvaire, structures céphaliques de la larve infestante, morphogenèse de ces structures du troisième stade de l'adulte, afin de préciser sa place zoologique dans l'évolution des Subuluridae.
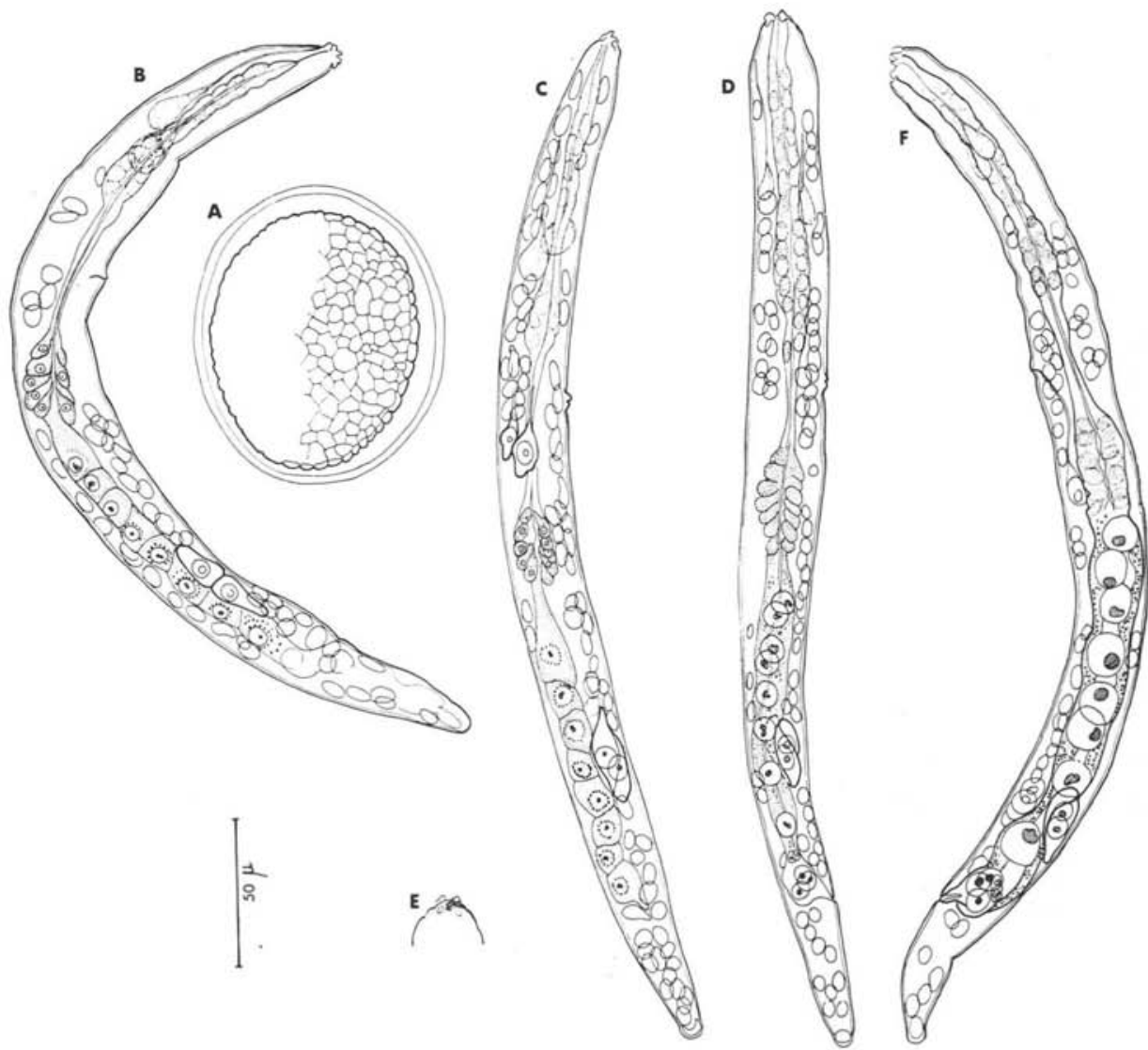

FIG. 2. - Tarsubulura perarmata. A: structure coquillière de l'œuf de $T$. perarmata; B et $\mathrm{C}$ : larves à l'éclosion; $\mathrm{D}$ : larve après $48 \mathrm{~h}$ de développement chez l'Insecte. On remarque la structure synticiale de l'intestin et l'apparition d'une lumière intestinale; E: détail de l'épine céphalique située sur la face ventrale de la larve; $\mathrm{F}$ : larve après cinq jours de développement chez l'insecte.

A, B, C, D, E, F : éch. $50 \mu$. 


\section{Cycle biologique.}

Les œufs embryonnés de ce Subulure (fig. 2, $A$ ) sont placés entre les pièces buccales de Criquets, achetés au marché chinois de Kuala Lumpur, et appartenant aux genres Valanga et Oxya*. Des larves du premier stade ont également été obtenues après quelques jours de développement chez Blatella germanica. Les insectes sont maintenus à une température de $28^{\circ} \mathrm{C}$.

\section{Allure du développement.}

Dès les premières heures qui suivent l'infestation, les larves pénètrent dans la portion postérieure de l'intestin moyen de l'insecte. Au second et troisième stades elles seront à l'intérieur de capsules de type hémocytaire situées en avant des tubes de Malpighi et accrochées à la paroi de l'intestin moyen.

\section{Chronologie des mues.}

Le développement de ce Subulure est lent; la première mue survient sur des larves âgées de 14 jours. Certaines larves âgées de trois semaines ébauchent leur deuxième mue. Les larves âgées de 28 jours sont toutes au troisième stade.

\section{Croissance larvaire.}

A l'éclosion, les larves mesurent 345-350 $\mu$ de long sur 25-30 $\mu$ de large. Elles atteignent $800 \mu$ de long sur $55 \mu$ de large à la fin du premier stade et $1400 \mu$ de long sur $105 \mu$ de large après trois semaines de développement à la fin du deuxième stade. Les larves infestantes sont fortement repliées sur elles-mêmes. Elles mesurent $1100 \mu$ de long sur $115 \mu$ de large.

\section{1) Organogenèse ET MORPHOlogie des STADES LARVAires.}

Premier stade.

L'organogenèse de $T$. perarmata au cours du premier stade est représentée sur la figure 2 .

A l'éclosion (fig. 2, B, C) les larves sont longues de $350 \mu$. Leurs autres dimensions sont les suivantes :

Largeur 25-30 $\mu$, longueur de la région cesophagienne 180-188 $\mu$; longueur des glandes céphaliques 70-85 $\mu$, pore excréteur situé à 112-123 $\mu$ de l'apex, longueur de l'intestin 120$140 \mu$; distance des deux cellules R 1 au pore anal $55 \mu$; longueur de la queue : $42 \mu$.

*Nous remercions le Dr Lee de l'Université de Malaya qui a bien voulu nous déterminer les deux genres de Criquets infestés. 
Vers 48 heures, les 8 cellules intestinales se fusionnent en un syncytium, la lumière de l'intestin et les trois cellules rectales se différencient. Après cinq jours de développement (fig. 2, F) la lumière intestinale s'est élargie, les cellules R1 se sont divisées et les glandes céphaliques se sont raccourcies.

Les dimensions de cette larve sont peu différentes de celles de la larve à l'éclosion.

Longueur 355-360 $\mu$; largeur 23-27 $\mu$; longueur de la région œsophagienne $165 \mu$; longueur des glandes céphaliques 46-85 $\mu$; pore excréteur situé à 100-105 $\mu$ de l'apex ; longueur de l'intestin $140-150 \mu$; distance des cellules $\mathrm{R} 1$ à l'anus $42 \mu$; longueur de la queue $42-47 \mu$.

La croissance larvaire démarre brusquement six jours après l'infestation. Les larves mesurent alors $385 \mu$ de long sur $34 \mu$ de large. Chez les larves âgées de huit jours (fig. 3, A), l'intestin et le rectum sont dilatés... Un méconium intestinal et un bouchon rectal (fig. 2, B) sont excrétés.

Les dimensions de ces larves sont :

Longueur $495-500 \mu$; largeur $50 \mu$; anneau nerveux et pore excréteur situés respectivement à 100 et $135 \mu$ de l'apex; longueur de l'œsophage $175 \mathrm{H}$; longueur de l'intestin $200 \mu$; longueur de la queue $80 \mu$.

A la fin du premier stade larvaire $12 \nmid 14$ jours (fig. 3, C) les ébauches larvaires n'ont pas évolué.

Longueur 700-800 $\mu$; largeur $55 \mu$; anneau nerveux et pore excréteur situés respectivement à $130-140 \mu$ et 165-182 $\mu$ de l'apex. Longueur de l'œsophage $390 \mu$; longueur de l'intestin 400-500 $\mu$; distance de l'ébauche à l'anus $200 \mu$; longueur de la queue $80 \mu$.

\section{Deuxième stade larvaire.}

Le deuxième stade larvaire (fig. 3, D) est caractérisé par la différenciation d'une petite capsule buccale (fig. 3, E), par l'apparition de valvules œsophagiennes et par la croissance de l'intestin qui se charge de réserve.

A la fin du deuxième stade larvaire, les larves ont doublé de taille. Leurs dimensions sont :

Longueur $1400 \mu$; largeur $105 \mu$; anneau nerveux et pore excréteur situés respectivement à $130 \mu$ et $170 \mu$ de l'apex ; longueur totale de l'œsophage $330 \mu$; dimensions du bulbe $50 \times 40 \mu$; longueur de l'intestin $950 \mu$; distance de l'ébauche génitale à la pointe caudale $560 \mu$; longueur de la queue $135 \mu$.

\section{Troisième stade larvaire.}

La larve est globuleuse (fig. $3, F$ ) et présente deux fines ailes latérales, une queue conique et pointue; l'intestin chargé de réserves est replié sur lui-même.

Ses dimensions sont les suivantes: 
Longueur mesurée sur le champ latéral $1100 \mu$; largeur $115 \mu$; anneau nerveux et pore excréteur situés à $85 \mu$ et $170 \mu$ de l'apex; longueur totale de l'œsophage $325 \mu$; dimensions du bulbe $52-45 \mu$; longueur de l'intestin $900 \mu$; ébauche génitale située à $520 \mu$ de l'extrémité caudale; longueur de la queue $95 \mu$.
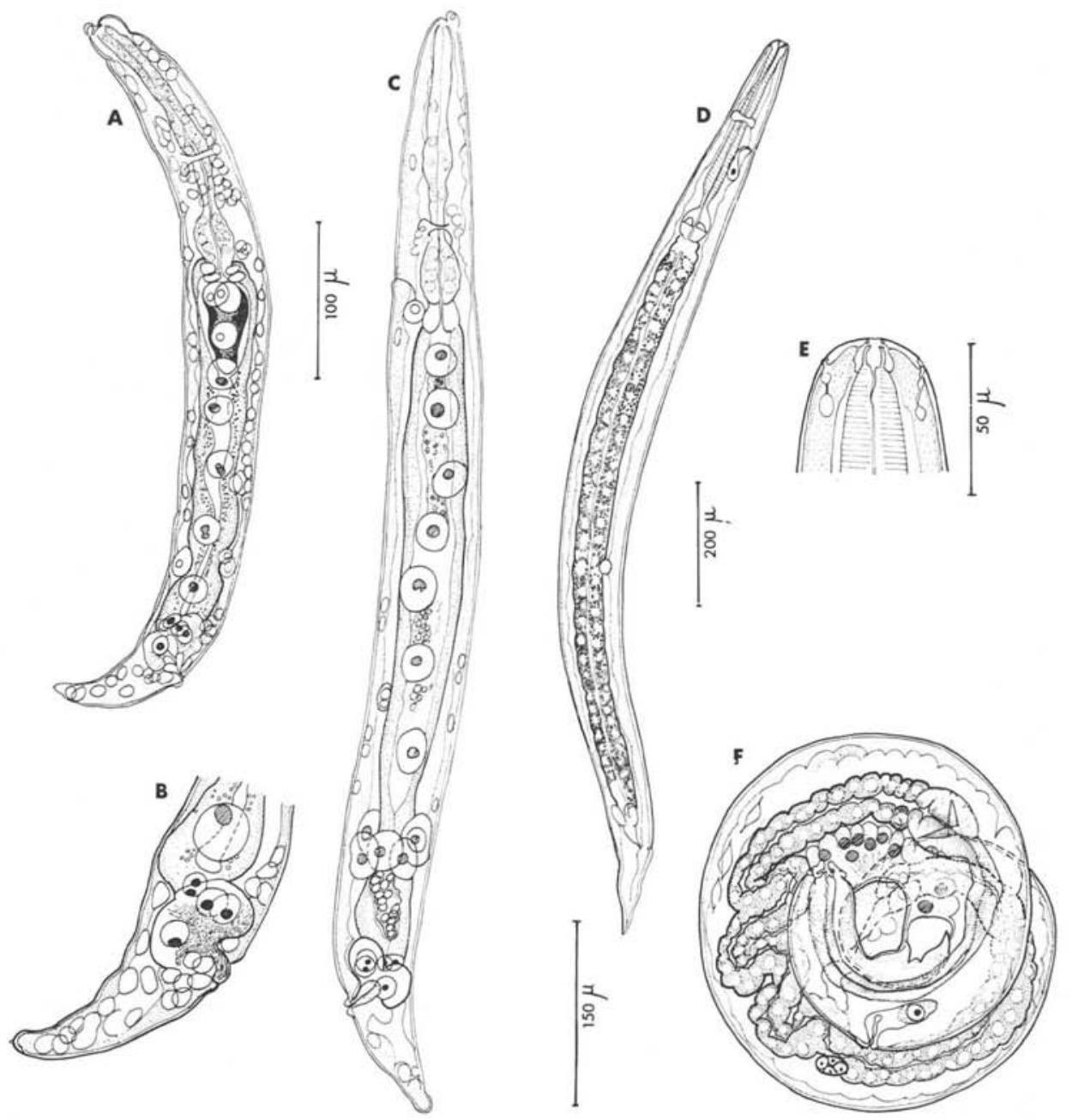

Fig. 3. - Tarsubulura perarmata. A : larve âgée de huit jours. On remarque le méconium excrété dans la lumière de l'intestin; B : détail de l'extrémité caudale et des cellules rectaies excrétant le bouchon rectal; C: larve âgée de 12 jours (fin du premier stade); D : larve du deuxième stade âgée de 3 semaines; $\mathrm{E}$ : détail de l'extrémité céphalique du deuxième stade larvaire; $\mathrm{F}$ : larve du troisième stade.

A. C : éch. $100 \mu$; B, E : éch. $50 \mu$; D : éch. $200 \mu$; F : éch. $150 \mu$. 


\section{Quatrième stade larvaire.}

La plus petite larve du quatrième stade résultant d'une infestation spontanée que nous avons pu récolter chez un Tupaia glis mesure $3 \mathrm{~mm}$ de long. Il s'agit d'une larve femelle dont les autres dimensions sont :

Largeur: $150 \mu$; anneau nerveux et pore excréteur situés à 170 et $260 \mu$ de l'apex ; profondeur du vestibule buccal $24 \mu$; longueur de l'œsophage $570 \mu$; dimensions du bulbe $120 \mu \times 100 \mu$; longueur de l'intestin $2200 \mu$; longueur de l'ébauche génitale: $530 \mu$. L'emplacement du futur ovéjecteur est situé à $630 \mu$ de l'anus; longueur de la queue $210 \mu$.

2) Ontogénèse des structures céphaliques du troisième stade a L'adulte. Structures céphaliQues du TROISIÈme STADE.

- Les structures céphaliques des larves infestantes (fig. 4 III, $A, B, C, D$ ) sont remarquables par l'apparition de trois épaisses formations cuticulaires pharyngiennes que nous identifions comme étant les ébauches de lobes périphériques. Les ébauches des lobes radiaux et chordaux ne sont encore représentées que sous forme de rides de la paroi pharyngienne comparables à celles visibles dans le genre Allopada (ct. Inglis, 1958-1964).

La cavité buccale est bordée par une paroi cuticulaire qui correspondra chez l'adulte à la première portion cuticulaire du vestibule buccal. La bouche est formée par six lobes labiaux terminés chacun par une papille du cycle labial interne. Les quatre papilles labiales externes sont volumineuses et accolées aux quatre papilles céphaliques.

Structures céphaliques du QUATRIÈme STADE (fig. 4, IV).

- Une transformation profonde s'opère au niveau des structures céphaliques du troisième au quatrième stade larvaire.

- La bouche s'ouvre et présente un contour hexagonal.

- La paroi cuticulaire qui constitue la première partie du vestibule buccal remonte du fait des poussées labiales et occupe sa position définitive.

FIG. 4. - Détail des structures céphaliques du troisième stade (III) et du quatrième stade (IV) de Tarsubulura perarmata.

III. Troisième stade larvaire: A : tête en vue ventrale. On remarque le développement des lobes périphériques (l.p.) cuticulaires; B : tête en vue latérale; C : tête en vue apicale.

Détail des 6 lobes labiaux. D: coupe optique au niveau des structures pharyngiennes.

$$
\text { A, B, C, D : éch. } 25 \mu \text {. }
$$

IV. Quatrième stade larvaire: A: tête en vue dorsale; B : tête en vue latérale gauche ; C: tête en vue apicale au niveau de la bouche; D : coupe optique au niveau de la capsúle buccale; E: coupe optique au niveau des structures pharyngiennes.

A, B, C, D, E: éch. $50 \mu$. 


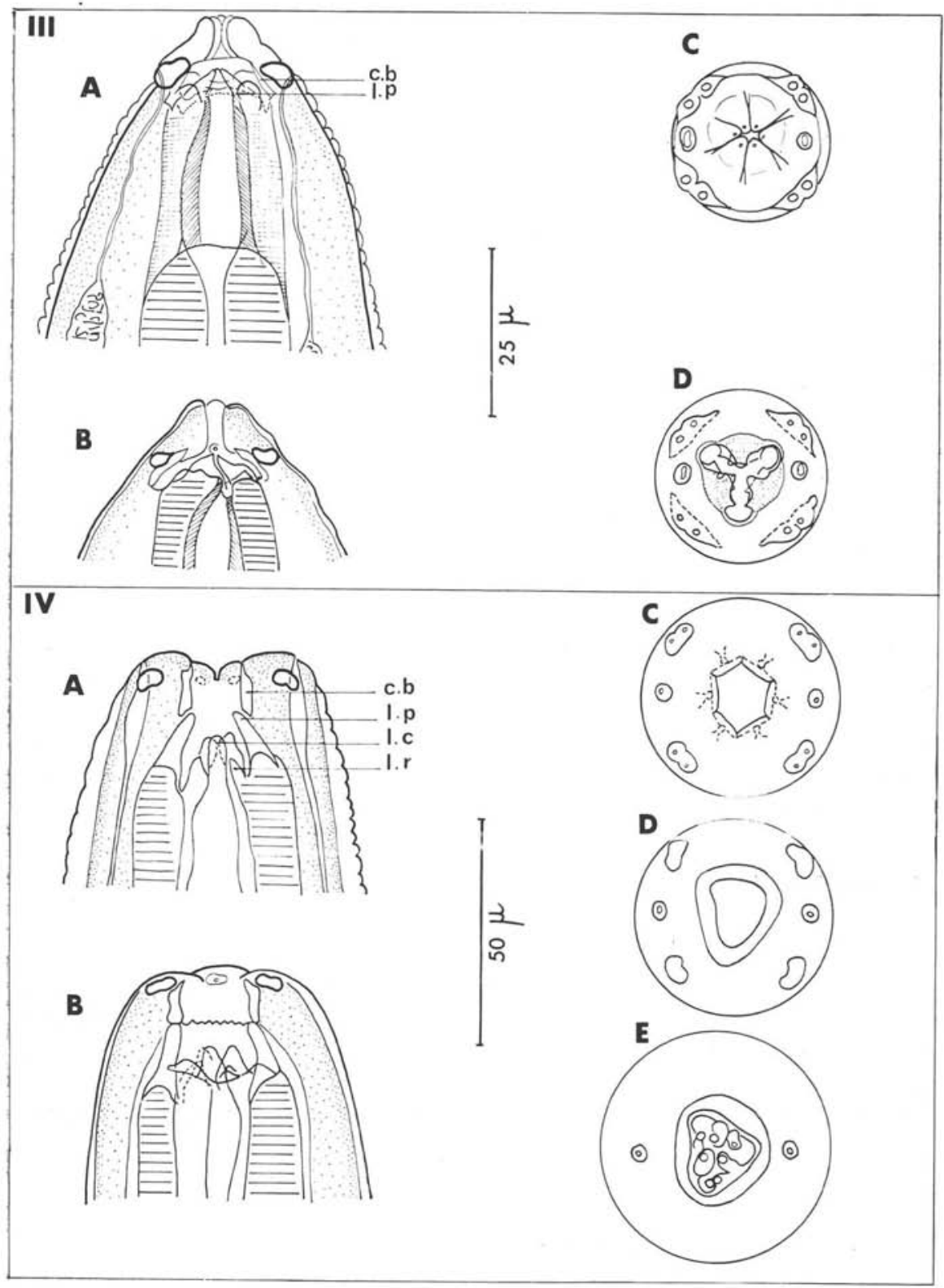


- Les lobes périphériques cuticulaires situés à l'extrémité de l'œsophage se redressent et forment en profondeur la paroi de la cavité pharyngienne (fig. $4 \mathrm{IV}$, A).

- Enfin, les lobes chordaux et radiaux cuticulaires nouvellement apparus à la base des lobes périphériques se dressent indépendamment dans la cavité buccale.

Aucune modification anatomique n'intervient du quatrième stade larvaire à l'adulte au niveau des structures céphaliques.

\section{Discussion}

Trois éléments présentent un intérêt phylogénique dans le cycle de Tarsubulura perarmata :

- son organogenèse ;

- sa croissance larvaire ;

- l'ontogenèse de ses structures céphaliques.

\section{Organogenèse.}

L'organisation interne de la larve à l'éclosion et la lente évolution des ébauches tissulaires au cours du premier stade et au début du second stade larvaires sont tout à fait identiques à l'organogenèse observée chez Allodapa suctoria par Quentin et Poinar, 1973.

Le nombre élevé d'initiales tissulaires chez la larve à l'éclosion (8 cellules intestinales en chapelet, 2 cellules $\mathrm{R}$ 1), et l'évolution lente de ces initiales au cours du premier stade et au début du second stade semble donc être un caractère commun à tous les Subuluridae.

\section{Croissance larvaire.}

Contrairement à ce que laissait présumer la morphologie très archaïque de l'adulte, la croissance larvaire de ce Subulure diffère de celle des Subulures primitifs du genre Allodapa, parasites d'Oiseaux et correspond à celle des Subulures parasites de Mammifères dont les cycles sont conmus (Subulura jacchi, Primasubulura distans, Subulura otolicni, Subulura williaminglisi). (Cf. Chabaud et Larivière, 1955 ; Petter, 1960 ; Quentin et Tchéprakoff, 1969 ; Quentin, 1969).

La larve, à l'éclosion, a en effet une taille (340-350 $\mu)$ inférieure à celle des larves d'Allopada mais la croissance ensuite est plus importante puisque la larve atteint $1100 \mu$ de long (moins de $500 \mu$ chez Allodapa).

\section{Ontogenèse des structures céphaliques.}

Cependant, les larves du troisième stade de Tarsubulura diffèrent de celles du genre Subulura par leurs structures céphaliques. Celles-ci possèdent, en effet, d'épais 
lobes périphériques cuticulaires absents sur les larves infestantes de Subulura ( $C f$. Quentin et Tchéprakoff, 1969) (Quentin, 1969).

L'apparition et la différenciation précoce au cours de l'ontogenèse céphalique de ces lobes périphériques cuticulaires qui sont une des particularités du genre Tarsubulura témoigne de l'isolement phylogénique de ce genre par rapport aux autres Subuluridae et justifie l'hypothèse d'Inglis, 1960, d'une lignée particulière au genre Tarsubulura.

Ce genre est primitif car il n'existe aucune différenciation des structures pharyngiennes et labiales du quatrième stade à l'adulte. La bouche reste béante sans lobes labiaux.

Dans la même lignée, le genre Labiobulura qui possède aussi des lobes pharyngiens cuticulaires mais qui présente en plus des lobes labiaux séparés chez certaines espèces par des interlabia, paraît représenter une nouvelle étape dans la différenciation des structures labiales.

Au sein de la sous-famille des Labiobulurinae, le genre Labiobulura parasite de Marsupiaux australiens apparaît ainsi plus évolué que le genre Tarsubulura parasite de Tupaidés, de Lorisiidés et de Tarsiidés asiatiques.

\section{Bibliographie}

BAYlis (H. A.), 1926. - Some parasitic worms from Sarawak. Sarawak Mus. J., 3, 303-322.

Baylis (H. A.), 1944. - Notes on some parasitic nematodes. Ann. Mag. nat. Hist., 11, 793-804.

Chabaud (A.-G.) et Larivière (M.), 1955. - Cycle évolutif d'un Ascaride: Subulura jacchi (Marcel, 1857) parasite de Primates, chez la Blatte Blabera fusca. C.R. Soc. Biol., 149, 1416-1419.

INGLIS (W. G.), 1958. - The comparative anatomy of the Subulurid head (Nematoda): with a consideration of its systematic importance. Proc. Zool. Soc. London., 130, 577-604.

INGLIS (W. G.), 1960. - Further observations on the comparative anatomy of the head in the family Subuluridae: with the description of new species. Proc. Zool. Soc. London, 135, 125-136.

INGLIS (W. G.), 1964. - Functional deformation in the head of the genus Allodapa (Nematoda). J. Helm., 38, 31-40.

INGLIS (W. C.), 1966. - The origin and function of the cheilostomal complex in the nematode Falcaustra stewarti. Proc. Linn. Soc. Lond., 177, 55-62.

KHERA (S.), 1956. - Nématode parasites from some Indian vertebrates. Indian J. Helminth., 6, 27-133.

MEYer (A.), 1896. - Neue ceylonische Nematoden aus Säugetieren (Filaria, Strongylus). und aus Julus (Oxyuris). Arch. Naturgesch., 62, 54-82.

Petter (A.-J.), 1960. - Sur une larve de Subuluridae parasite de la Blatte germanique (Blatella germanica L.). C.R. Soc. Biol., 154, 300-301. 
Quentin (J.-C.), 1969. - Cycle biologique de Subulura williaminglisi Quentin, 1965. Ontogenèse des structures céphaliques. Valeur phylogénétique de ce caractère dans la classification des Nématodes Subuluridae. Ann. Parasitol. hum. comp., 44, 451484.

Quentin (J.-C.) et Poinar (G. O.), 1973. - Comparative study of the larval development of some heteroxenous subulurid and spirurid nematodes. Intern. J. Parasit., 3, 809-827.

QUENTIN (J.-C.) et TChePraKofF (R.), 1969. - Cycle biologique de Subulura otolicni (Van Beneden, 1890). Bull. Mus. Nat. Hist. Nat., $2^{\circ}$ sér., 41, 571-578.

RatZel (F.), 1868. - Beschreibung einiger neuen Parasiten. Arch. Naturgesch., 34, 150156. 